\title{
Alterações nos níveis de colesterol, triglicerídeo e fosfolipídeo total em plasma de Callithrix jacchus (saguii) reinfectado por Schistosoma mansoni
}

\author{
Alterations in cholesterol, triglyceride and total phospholipid levels in plasma \\ of Callithrix jacchus (sagüi) reinfected by Schistosoma mansoni
}

\author{
Thadzia Maria de Brito Ramos $\quad{ }^{1}$, Amanda Soares de Vasconcelos $\quad{ }^{1}$, Vera Cristina \\ Oliveira de Carvalho ${ }^{1}$ e Vera Lúcia de Menezes Lima ${ }^{1}$
}

\section{RESUMO}

Pouco se conhece a cerca de alterações nos lipídeos plasmáticos devido à reinfecção por Schistosoma mansoni. Neste trabalho, foram avaliadas alterações nos lipideos plasmáticos decorrentes de uma reinfecção por Schistosoma mansoni no primata não bumano Callithrix jacchus (sagüi). Amostras de sangue dos animais, antes e após serem infectados e reinfectados, foram coletadas por punção venosa, anticoaguladas com EDTA $(1 \mathrm{mg} / \mathrm{mL})$ e centrifugadas a 2.500xg para obtenção do plasma. Os níveis plasmáticos de colesterol total, colesterol éster, fosfolipideo total e triglicerideo foram determinados por métodos espectrofotométricos. Os resultados mostraram haver redução significativa nas concentrações de colesterol total, colesterol esterificado, triglicerídeo e fosfolipideo total em plasma de animais reinfectados por Schistosoma mansoni, em comparação com os mesmos animais antes da infecção e após uma infecção. Este estudo mostra que uma segunda infecção por Schistosoma mansoni causa alterações lipídicas plasmáticas significativamente mais acentuadas que as decorrentes de uma única infecção.

Palavras-chaves: Callithrix jacchus. Schistosoma mansoni. Reinfecção. Colesterol. Triglicerídeo.

\section{ABSTRACT}

Little information is available on the lipid changes caused by Schistosoma mansoni reinfection. In this work it was evaluated alteration in the plasma lipids due to one reinfection by Schistosoma mansoni in the non buman primate Callithrix jacchus (sagüi). Blood samples from C. jacchus, prior and after 60 days infection and reinfection, were collected by intravenous puncture, anticoagulated with EDTA $(1 \mathrm{mg} / \mathrm{mL})$ and centrifuged at 2,500xg, in order to obtain the plasma. Total cholesterol, cholesteryl ester, total phospholipid and triglyceride levels were determined by spectrophotometer methods. The results showed that there are significant reduction in cholesterol total, cholesteryl ester, total phospholipid and triglyceride concentrations in plasma of animals reinfected by Schistosoma mansoni, in comparison to the same animals prior and after one infection. This study showed that a second infection of Callithrix jacchus by Schistosoma mansoni causes plasma lipid alterations, which are more significant than after a single infection. Key-words: Callithrix jacchus. Schistosoma mansoni. Reinfection. Cholesterol. Triglycerides.

A esquistossomose é uma das maiores causas de morbidade e mortalidade no mundo $0^{5}$ atingindo milhões de pessoas em países da África e América Latina. No Nordeste do Brasil, a doença é endêmica, atingindo áreas rurais e urbanas, bem como, tem sido constituindo novos focos de transmissão também em localidades de veraneio ${ }^{3}$. As falhas na erradicação do molusco vetor ${ }^{29}$, a inexistência de vacina para a prevenção da doença, além da recente resistência desenvolvida pelo parasita frente às drogas anti-esquistossomóticas ${ }^{16}$ constituem algumas das dificuldades para o controle da doença, que vem permanecendo, ainda, como um importante problema médico-social².

A parasitemia humana causada pelo Schistosoma mansoni representa um ciclo contínuo de tratamento seguido de reinfecção. A morbidade da esquistossomose mansônica depende da intensidade da infecção, resposta imune, reação granulomatosa e conseqüente fibrose em torno dos ovos de Schistosoma mansoni depositados no

\footnotetext{
1. Laboratório de Química e Metabolismo de Lipídeos e Lipoproteínas do Centro de Ciências Biológicas da Universidade Federal de Pernambuco, Recife, PE. Apoio Financeiro: CNPq, FACEPE, BNB e CAPES

Endereço para correspondência : Prof ${ }^{a}$ Vera Lúcia de Menezes Lima. Dept ${ }^{\circ}$ Bioquímica/CCB/UFPE. Av. Prof. Morais Rego s/n, Cidade Universitária, 50670-420 Recife, PE. Tel: 81 3271-8540; Fax: 81 3271-8576;

e-mail:vlml@ufpe.br

Recebido para publicação em 13/8/2002

Aceito em 13/11/2003
} 
tecido do hospedeiro ${ }^{23}$. Os danos teciduais afetam órgão vitais como o intestino e o fígado, podendo alterar processos metabólicos e afetar as condições físicas do hospedeiro ${ }^{22}$. A esquistossomose na forma hepatoesplênica pode vir associada a varizes hemorrágicas do esôfago, hiperesplenismo e hipoevolutismo (em adolescentes), podendo a evolução clínica desses pacientes apresentar-se comprometida por carga parasitária residual ou reinfecção $0^{6}$.

Estudos anteriores em humanos portadores de esquistossomose mansônica na forma hepatoesplênica demonstram a ocorrência de alterações no metabolismo lipídico associadas à esquistossomose mansônica, na qual a concentração plasmática de colesterol total encontra-se diminuída, bem como os níveis de fosfolipídeo e triglicerídeo ${ }^{11}$, além da fração esterificada do colesterol ${ }^{7}$. A utilização de um modelo animal no qual a deslipidemia, causada unicamente pela esquistossomose mansônica, possa ser estudada sem associação com desnutrição ou outras parasitoses comumente encontradas em humanos portadores de esquistossomose mansônica é de extrema importância para um melhor conhecimento da fisiopatologia da doença. Atualmente, primatas não-humanos vêm sendo usados para diversos estudos ${ }^{1}$ inclusive aqueles envolvendo a migração de $S$. mansoni pelo hospedeiro definitivo ${ }^{31}$. Trabalhos publicados anteriormente demonstram que o Callithrix jacchus (sagüi), primata do novo mundo, animal de pequeno porte e comumente encontrado na América do Sul, apresenta a composição lipídica e das proteínas plasmáticas bastante similares àquelas encontradas em plasma de humanos ${ }^{20}$. No presente trabalho foram avaliados os efeitos da reinfecção pelo parasita S. mansoni sobre os lipídeos plasmáticos de Callithrix jacchus.

\section{MATERIAL E MÉTODOS}

Animais. No presente estudo foram utilizados 11 Callithrix jacchus, machos adultos, pesando $300 \mathrm{a}$ 400g. Os animais foram cedidos pelo Instituto Brasileiro do Meio Ambiente (IBAMA) e eram aqueles apreendidos em feiras publicas que não podiam ser reintroduzidos ao seu ambiente natural. Os animais foram mantidos no biotério do Departamento de Bioquímica da UFPE, onde permaneceram em quarentena antes do uso, sob condições adequadas de higiene e dieta ${ }^{8}$. A presença de parasitas nas fezes e no sangue dos animais foi investigada e foram utilizados apenas animais saudáveis, livres de qualquer parasitose detectada.

Infecção. Os animais foram percutaneamente infectados ${ }^{26} \mathrm{com}$ cerca de 400 cercárias de Schistosoma mansoni, ficando expostos à luz $\left(35^{\circ} \mathrm{C}\right)$ por aproximadamente $35 \mathrm{~min}$ para a penetração das mesmas, sob anestesia com cloridrato de cetamina - Ketalar (Parke Davis Ache/São Paulo Brasil) na concentração de $15 \mathrm{mg} / \mathrm{kg}$ de peso corpóreo.

Re-infecção. 60 dias após a infecção os animais foram novamente anestesiados com Ketalar $(15 \mathrm{mg} / \mathrm{kg})$ e submetidos a uma segunda infecção percutânea com aproximadamente 400 cercárias de $S$. mansoni, nas condições de temperatura e luminosidade acima descritas.

Obtenção do plasma. Amostras de sangue de animais, em jejum de 12h, foram coletadas antes (grupo controle), após 60 dias da primeira (grupo infectado), e após 60 dias da segunda infecções (grupo reinfectado), em animais anestesiados, por punção da veia femoral. As amostras foram anticoaguladas com EDTA (1mg/mL) e centrifugadas a $2.500 \mathrm{xg}, \mathrm{a} 4^{\circ} \mathrm{C}$, durante $15 \mathrm{~min}$, para a obtenção dos plasmas.

Extração, isolamento e determinação dos níveis de lipídeos plasmáticos. Os lipídeos plasmáticos foram extraídos com clorofórmio:metanol 2:1 (v/v) de acordo com o método de Folch ${ }^{10}$. 0 isolamento dos lipídeos neutros foi realizado por cromatografia em camada fina ${ }^{21}$, sendo em seguida removidos da sílica e processados como descrito em trabalho anterior ${ }^{11}$. A determinação dos níveis de colesterol total, e esterificado no plasma de C. jacchus controles, infectados e reinfectados foi realizada segundo o método de Leffler ${ }^{17}$. A concentração de fosfolipídeo total foi determinada de acordo com o método de Bartlett ${ }^{4} \mathrm{e}$ a análise de triglicerídeo foi realizada através de método enzimático/colorimétrico utilizando kit comercial Labtest Diagnóstica (Minas Gerais, Brasil).

Tratamento estatístico dos resultados. Os resultados representam a média dos valores encontrados \pm 0 desvio padrão. A significância das diferenças entre os grupos de animais controles, infectados e reinfectados foi determinada pelo teste " $\mathrm{t}$ " de Student para análises dependentes, utilizando o software STATISTICA for Windows (StatSoft, Inc, 1999 - Tulsa, USA). Diferenças significativas entre os grupos foram estabelecidas considerando $\mathrm{p}<0,05$.

\section{RESULTADOS}

Os níveis de colesterol total, triglicerídeos e fosfolipídio total em plasma de $C$. jacchus após 60 dias da primeira infecção por $S$. mansoni foram significativamente reduzidos $(\mathrm{p}=0,005 ; \mathrm{p}=$ 0,$000048 ; p=0,000042$, respectivamente) em relação aos animais controles, ou seja, antes da primeira infecção. Essa redução foi ainda mais significativa quando os animais foram submetidos a uma segunda infecção pelo parasita $S$. mansoni, tanto para a concentração de colesterol total $(\mathrm{p}=0,0005)$, quanto para a de triglicerídeos $(p=0,000007)$ e de fosfolipídio total $(p=0,000000)$, se comparados aos animais controle, conforme demonstrado na Tabela 1.

Embora os níveis de colesterol total e fosfolipídio total tenham sido reduzidos a relação molar colesterol total/fosfolipídio total foi significativamente aumentada ( $\mathrm{p}=0,000802)$ em plasma de sagüis infectados em relação aos animais controles, tendo sido este aumento mais significativo após a reinfecção $(\mathrm{p}=0,000324)$ quando comparado aos animais controle, como demonstrado na Tabela 1.

A percentagem de colesterol esterificado foi significativamente reduzida ( $\mathrm{p}=0,000128)$ em plasma de sagüis infectados por Schistosoma mansoni, quando comparados aos animais controles; após a reinfecção esta redução em relação aos animais controle foi ainda mais significativa $(\mathrm{p}=0,000007)$, como demonstrado na Figura 1.

Na Tabela 1, podemos observar, também, que em comparação aos resultados obtidos para os lipídeos plasmáticos de animais submetidos à primeira infecção, a segunda infecção promoveu reduções significativas nos níveis de colesterol total $(p=0,040831)$, 
Tabela 1 - Concentração plasmática de colesterol total, triglicerídeo e fosfolipídio total em Callithrix jacchus reinfectado por Schistosoma mansoni.

\begin{tabular}{|c|c|c|c|c|}
\hline \multirow[b]{2}{*}{ Grupos } & \multicolumn{3}{|c|}{ Lipídeos plasmáticos (mmol/L) } & \multirow{2}{*}{$\begin{array}{l}\text { Colesterol total/ } \\
\text { fosfolipídio total }(\mathrm{mol} / \mathrm{mol})\end{array}$} \\
\hline & colesterol total & triglicerídeos & fosfolipídio total & \\
\hline Não infectados & $2,81 \pm 0,14$ & $1,35 \pm 0,22$ & $2,18 \pm 0,17$ & $1,29 \pm 0,13$ \\
\hline Infectados & $2,67 \pm 0,14^{* 2}$ & $1,31 \pm 0,22^{* 5}$ & $1,97 \pm 0,15^{* 5}$ & $1,36 \pm 0,12^{* 3}$ \\
\hline Reinfectados & $2,60 \pm 0,15^{* * * 4, \cdots * * * 1}$ & 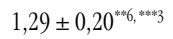 & $1,79 \pm 0,14^{* * 6,6 * w 4}$ & $1,46 \pm 0,14^{* * w_{4} * * * * 1}$ \\
\hline
\end{tabular}

Os valores representam a média \pm 0 desvio padrão.

"Diferença significativa entre os grupos controle e infectados. "*** Diferença significativa entre os grupos controle e reinfectados. ${ }^{* * *}$ Diferença significativa entre os grupos infectados e reinfectados. ${ }^{1} \mathrm{p}<0,05 ;{ }^{2} \mathrm{p}<0,005 ;{ }^{3} \mathrm{p}<0,001 ;{ }^{4} \mathrm{p}<0,0005 ;{ }^{5} \mathrm{p}<0,00005 ;{ }^{6} \mathrm{p}<0,00001$

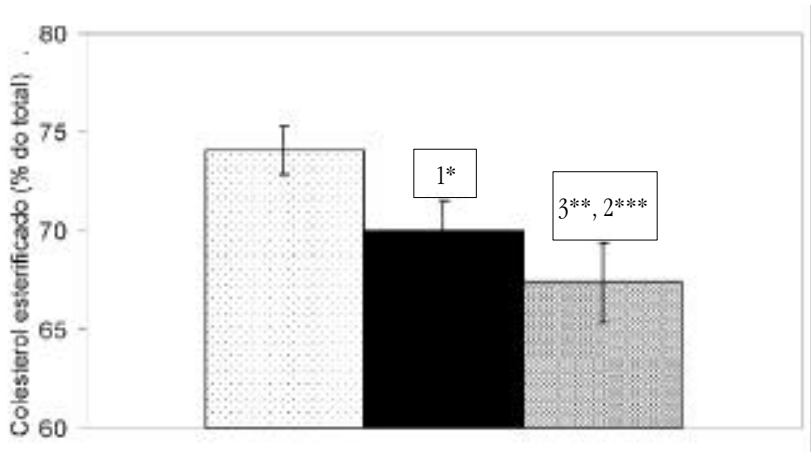

Figura 1 - Concentração relativa de colesterol éster em plasma de Callithrix jacchus reinfectado por Schistosoma mansoni.

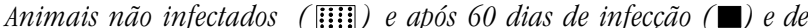
reinfecção ( ) por Schistosoma mansoni. Diferença significativa entre os grupos: "controle e infectados, *controle e reinfectados, e, ***infectados e reinfectados, de $10<0,0001 ;{ }^{2} p<0,0005 e^{3} p<0,00001$.

triglicerídeo $(\mathrm{p}=0,001719)$ e fosfolipídio total $(\mathrm{p}=0,000523)$, bem como na percentagem de colesterol esterificado $(\mathrm{p}=0,00041)$ demonstrada na Figura 1. A relação molar colesterol total/fosfolipídio total (Tabela 1) foi significativamente aumentada $(\mathrm{p}=0,007375)$ no plasma de sagüis submetidos à segunda infecção por S. mansoni, quando comparada aos valores obtidos para a primeira infecção (Tabela 1).

\section{DISCUSSÃO}

Em trabalhos anteriores foi demonstrado que o C. jacchus apresenta similaridade ao homem no que diz respeito à composição dos lipídeos e lipoproteínas plasmáticas ${ }^{20}$, e que o fígado deste primata está histopatologicamente suscetível a infecção por Schistosoma mansoni ${ }^{26}$.

A redução nos valores encontrados, no presente trabalho, para os níveis de triglicerídeo, colesterol e fosfolipídio total (Tabela 1 e Figura 1) após 60 dias de infecção de C. jacchus por S. mansoni foram similares aos reportados anteriormente por Lima $\mathrm{et} l^{20}$. No plasma de $C$. jacchus saudável o nível de colesterol total e a concentração relativa de colesterol esterificado reportadas por Lima

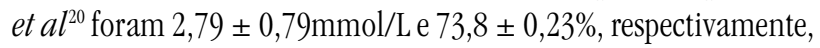
porém, quando infectados por cercarias de $S$. mansoni o colesterol total e a percentagem de colesterol esterificado sofreram reduções significativas para 2,35 $\pm 0,32 \mathrm{mmol} / \mathrm{L}$ e $65,9 \pm 3,9 \%$, respectivamente. Em humanos portadores de esquistossomose mansônica ${ }^{7}$ a redução do colesterol foi associada à gravidade da doença, sendo mais suave na esquistossomose hepatoesplênica compensada do que na forma hepatoesplênica descompensada ${ }^{13}$. Estudos realizados tanto em humanos ${ }^{2718}$ quanto experimentalmente em roedores ${ }^{2428}$ sugerem que o decréscimo na fração esterificada do colesterol está relacionado à diminuição na atividade de enzimas de origem hepática. Por exemplo, a lecitina: colesterol aciltransferase (LCAT, EC 2.3.1.43) plasmática, sintetizada pelos hepatócitos ${ }^{19} \mathrm{e}$ secretada na circulação, atua catalizando a esterificação do colesterol na superfície da lipoproteína de alta densidade $(\mathrm{HDL})^{15}$ plasmática, promovendo o efluxo de colesterol dos tecidos periféricos ${ }^{9}$, enquanto a acil CoA colesterol aciltransferase (ACAT, EC 2.3.1.26) é responsável pela esterificação de colesterol no fígado. Portanto, futuros estudos são necessários para avaliar se a redução nos níveis de colesterol esterificado plasmático de $C$. jacchus é decorrente de anormalidade na atividade, na síntese ou na secreção da LCAT e/ou da ACAT produzidas pelo fígado doente dos animais reinfectados.

Os lipídeos neutros e fosfolipídeos são transportados na circulação pelas lipoproteínas, as quais em contato com membranas celulares trocam lipídeos entre $\mathrm{si}^{25}$. Neste trabalho, o aumento significativo na relação molar colesterol total/fosfolipídio total em Callithrix jacchus infectados e reinfectados parece estar mais relacionado ao decréscimo nos níveis de fosfolipídeos plasmáticos desses animais, do que ao do colesterol total. Alterações na composição dos lipídeos circulantes podem promover mudanças na composição dos lipídeos de membranas. Modificação na composição lipídica de membrana celular pode levar a alteração na função celular como demonstrado em trabalhos anteriores ${ }^{14}$. Em experimentos realizados com macaco foi demonstrado que alteração nos fosfolipídeos de HDL modifica o tipo de éster de colesterol formado no plasma afetando a quantidade plasmática de colesterol esterificado $0^{30}$. Variação na relação molar colesterol total/fosfolipídio total foi reportadas na literatura para pacientes portadores de esquistossomose hepatoesplênica ${ }^{11}$.

Em relação à primeira infecção, as diminuições significativas observadas nos níveis dos lipídeos plasmáticos de animais reinfectados implicam em um efeito cumulativo sobre o metabolismo lipídico de C. jacchus promovido pela reinfecção parasitária pelo $S$. mansoni. Alterações significativas nos lipídeos circulantes decorrentes apenas de reinfecção por $S$. mansoni podem estar associadas a mudanças na fluidez de membranas plasmáticas e/ou na função celular contribuindo para a patologia da doença. Imai e 0 wen $^{14}$ demonstraram, experimentalmente, in vitro, que a função celular era alterada quando a composição lipídica da membrana celular estava modificada. Portanto, os achados deste estudo são importantes para o conhecimento da fisiopatologia da esquistossomose mansônica com relação às alterações no metabolismo lipídico em primata ${ }^{71220}$. Este trabalho também reforça a proposta de utilização de $C$. jacchus em pesquisas que promovam maior conhecimento sobre a fisiopatologia causada por infecções 
sucessivas pelo $S$. mansoni, tendo como alvo avaliação do metabolismo lipídico associado, por exemplo, à análise da eficácia de novas drogas anti-esquistossomóticas, vacinas e desenvolvimento de novos testes para diagnósticos laboratoriais da doença, haja vista que este primata apresenta base bioquímica segura para estudos envolvendo alterações no metabolismo lipídico na esquistossomose experimental.

\section{AGRADECIMENTOS}

Agradecemos à Dra. Geni Vilela da Silva Carvalho (IBAMA) pela disponibilização dos animais, à Dra. Silvia Montenegro Chefe do Departamento de Imunologia - CPqAM/FIOCRUZ por ter cedido as cercárias de $S$. mansoni pra infecção de C. jacchus, e ao CNPq, FACEPE, BNB e CAPES pelo suporte financeiro.

\section{REFERÊNCIAS BIBLIOGRÁFICAS}

1. Andrade MCR, Oliveira NA, Romijn PC. Resposta imune produzida por vacinas anti-rábicas em sagüis (Callithrix sp). Revista da Sociedade de Medicina Tropical 32: 533-240, 1999.

2. Barbosa CS. Epidemiology and anthropology: an integrated approach dealing with bio-socio-cultural aspects as strategy for the control of endemic diseases. Memórias do instituto Oswaldo Cruz 93: 59-62, 1998.

3. Barbosa CS, Silva CB, Barbosa FS. Esquistossomose: reprodução e expansão da endemia no Estado de Pernambuco no Brasil. Revista de Saúde Pública 30: 609-616, 1996.

4. Bartlett GR. Phosphorus assay in column chromatography. Journal of Biological Chemistry 243: 466-468, 1959 .

5. Bica I, Hamer DH, Stadecker MJ. Hepatic schistosomiasis. Infectious Disease Clinics of North America 14: 583-604, 2000.

6. Brandt CT, Sá HP, Caneca OAF, Santana JV, Miranda P, Carvalheira R. Esquistossomose hepatoesplênica em adolescentes: carga parasitaria residual após tratamento clínico-cirúrgico. Anais da Faculdade de Medicina da Universidade de Pernambuco 43: 123-126,1998.

7. Chechinel YMN, Owen JS. 0 colesterol plasmático na esquistossomose mansônica. Revista Brasileira de Biologia 38: 339-343, 1978.

8. Clapp NK, Tardif SD. Marmoset husbandry and nutrition. Digestive Diseases and Sciences 30: 17S-23S, 1985

9. Dobiasova M, Frohlich JJ. Advances in understanding of the role of lecithin:cholesterol acyltransferase (LCAT) in cholesterol transport. Clinica Chimica Acta 286: 257-271, 1999 .

10. Folch J, Lees M, Sloane-stanley GH. A simple method for the isolation and purification of total lipids from animal tissue. Journal of Biological Chemistry 226: 497-509, 1957.

11. Gillett MPT, Lima VLM, Costa JCM, Sibrian AM. Plasma lipid concentrations for some Brazilian lizards. Comparative Biochemistry and Physiology 64B: 273-277, 1979.

12. Gillett MPT, Oliveira MA. Altered erythrocyte and platelet lipid compositions in relation to lecithin: cholesterol acyltransfer deficiency in hepatosplenic schistosomiasis mansoni. Arquivos de Biologia e Tecnologia 27: 511-520, 1984.
13. Gillett MPT, Owen JS, Coutinho A. Plasma free and esterified cholesterol in schistosomiasis mansoni. The Lancet 2: 971, 1976.

14. Imai Y, Owen JS. The cholesterol content of renal cortical brush-border membranes affects $\mathrm{Na}^{+}$-dependent glucose transport. Biochemical Siciety Transactions 17: 881-882. 1989

15. Jonas A. Lecithin: cholesterol acyltransferase. Biochimica et Biophysica Acta 1529: 245-256, 2000.

16. Kalife J, Cêtre C, Pierrot C, Capron M. Mechanisms of resistance to S. mansoni infection: the rat model. Parasitology International 49: 339-345, 2000.

17. Leffler HH. Method for cholesterol and cholesteryl esters in serum. In: Sunderman FW, Sunderman FW Jr (ed) Lipids and Steroid Hormones in Clinical Medicine, Lippincott, Philadelphia, p. 18-22, 1960.

18. Lima VLM, Cannizzaro HM, Owen JS. Isolation and preliminary microheterogeneity studies of lecithin-cholesterol acyltransferase in plasma from individual patients infected with Schistosomiasis mansoni. International Hepatology Communications 6: 300-305, 1997.

19. Lima VLM, McIntyre N, Owen JS. Secretion of lecithin: cholesterol acyltransferase (LCAT) by the human cell line, Hep G2. Biochemical Society Transactions 16: $155-156,1988$

20. Lima VLM, Sena VLM, Stewart B, Owen JS, Dolphin PJ. An evaluation of Callithrix jacchus (sagüi) as an experimental model for the dyslipoproteinemia of human schistosomiasis mansoni. Biochimica et Biophysica Acta 1393: 235-243, 1998.

21. Mangold HK. Thin Layer Chromatography, Spring-Verlag. New York, 1969.

22. Muller E, Brunet LR, Fried B, Sherma J. Effects on the neutral lipid contents of the liver, ileum and serum during experimental schistosomiasis. International Journal for Parasitology 31: 285-287, 2001.

23. Ouma JH, Vennervaldb BJ, Kariukia HC, Butterworthc AE. Morbidity in schistosomiasis: an update. Trends in Parasitology 17: 117-118, 2001.

24. Owen JS, Costa JCM, Carvalho VCO, Gillett MPT. Effect of experimental schistosomiasis mansoni on plasma and erythrocyte lipids and on plasma lecithin: cholesterol acyltransferase. Revista Brasileira de Biologia 38: 913-918, 1978.

25. Owen JS, Gillett MPT, McIntyre N. Lipoproteins, cell membranes and cellular functions. Trends in Biochemical Sciences 9: 238-242, 1984

26. Santana JV, Figuerêdo-Silva J, Amâncio FF, Gonçalves JF, Malagueño E, Murfin DJ, Damian RT. Experimental schistosomiasis in Callithrix jacchus. A histopatologic study. Memórias do Instituto Oswaldo Cruz 84: 257, 1989.

27. Silva CA, Oliveira KF, Carvalho VCO, Domingues ALC, Brandt CT, Lima VLM. Efeito de tratamento cirúrgico sobre a atividade da enzima hepática lecitina: colesterol aciltransferase (LCAT) na esquistossomose mansônica. Acta Cirúrgica Brasileira 17: 28-30, 2001

28. Soares MGCB, Borges SS. Effect of experimental schistosomiasis on ${ }^{14} \mathrm{C}-4$ cholesterol esterification by mouse liver homogenate. Brazilian Journal of Medical and Biological Research 23: 7-9, 1990 .

29. Struck RF. Schistosomiasis epidemiology and control: How did we get and where should we go? Memórias do instituto Oswaldo Cruz 96: 17-27, 2001.

30. Thornburg JT, Parks JS, Rudel LL. Dietary fatty acid modification of HDL phospholipid molecular species alters lecithin: cholesterol acyltransferase reactivity in cynomolgus monkeys. Journal Lipid Research 36: 277-289, 1995.

31. Wilson RA, Coulson PS, Sturrock RF, Reid GDF. Schistosome migration in primates: a study in the olive babboon (Papio amubis). Transactions of the Royal Society of Tropical Medicine and Hygiene 84: 80-83, 1990. 